\title{
Predictors and prognosis of left ventricular thrombus in post- myocardial infarction patients with left ventricular dysfunction after percutaneous coronary intervention
}

\author{
Jieyun You $^{1 \#}$, Xingxu Wang ${ }^{1 \#}$, Jian $\mathrm{Wu}^{2 \#}$, Liming Gao ${ }^{1 \#}$, Xiaoyan Wang ${ }^{2}$, Peizhao Du ${ }^{1}$, Haibo Liu ${ }^{1}$, Jiming \\ Li $^{1}$, Yunkai Wang ${ }^{1}$, Yulu Liang ${ }^{1}$, Wei Guo ${ }^{1}$, Qi Zhang ${ }^{1}$ \\ ${ }^{1}$ Department of Cardiovascular Medicine, Shanghai East Hospital, Tongji University School of Medicine, Shanghai 200120, China; ${ }^{2}$ Shanghai \\ Institute of Cardiovascular Diseases, Zhongshan Hospital and Institutes of Biomedical Sciences, Fudan University, Shanghai 200032, China \\ Contributions: (I) Conception and design: Q Zhang, J You, X Wang, J Wu, L Gao; (II) Administrative support: Q Zhang; (III) Provision of study \\ materials or patients: Q Zhang, J You, X Wang, L Gao, H Liu, J Li, Y Wang, Y Liang, W Guo; (IV) Collection and assembly of data: J You, X Wang, \\ J Wu, X Wang, P Du; (V) Data analysis and interpretation: Q Zhang, J You, X Wang, J Wu, L Gao; (VI) Manuscript writing: All authors; (VII) Final \\ approval of manuscript: All authors. \\ "These authors contributed equally to this work. \\ Correspondence to: Qi Zhang. Department of Cardiovascular Medicine, Shanghai East Hospital, Tongji University School of Medicine, Shanghai \\ 200120, China. Email: zhangqnh@hotmail.com.
}

Background: We aimed to investigate the predictors and prognosis of left ventricular thrombus (LVT) in patients admitted for post-myocardial infarction (MI) and left ventricular dysfunction after contemporary percutaneous coronary intervention (PCI).

Methods: We prospectively enrolled 267 consecutive post-MI patients with left ventricular ejection fraction $(\mathrm{LVEF})<0.45$ based on the Shanghai East Hospital PCI database since 2012. Altogether 25 (9.36\%) patients were selected as the LVT group. Baseline, angiographic, procedural characteristics and 1-year clinical outcomes were compared by Chi-square test, $t$-test or Kaplan-Meier survival analysis as appropriate. Receiver operating characteristic (ROC) curves were plotted for the accuracy of the multivariate analysis model. A multiple logistic regression was applied to predict LV'T formation.

Results: The independent risk factors of LVT were left ventricular aneurysm [odds ratio (OR): 1.29, 95\% confidence interval (CI): 1.09-1.52, $\mathrm{P}<0.01$ ], incomplete revascularization (OR: 0.05, 95\% CI: 0.01-0.35, $\mathrm{P}<0.01$ ), SYNTAX score (OR: 1.28, 95\% CI: 1.14-1.43, P<0.01) and D-dimer (OR: 1.90, 95\% CI: 1.19-3.04, $\mathrm{P}<0.01)$. The SYNTAX score and D-dimer effectively indicated the development of LVT with optimal cutoff values of 29.50 and $1.53 \mathrm{mg} / \mathrm{L}$, respectively. Patients with LVT had significantly worse outcomes at 1-year clinical follow-up, especially higher incidence of ischemic or hemorrhagic stroke.

Conclusions: This study indicated that the presence of left ventricular aneurysm, incomplete revascularization, higher SYNTAX score and D-dimer level were the independent predictors of LVT formation in post-MI and LV dysfunction patients, which related to worse clinical outcomes. Future studies for early intervention and complete revascularization in high-risk subgroup patients are expected.

Keywords: Left ventricular thrombus (LVT); myocardial infarction (MI); left ventricular dysfunction; percutaneous coronary intervention (PCI)

Submitted May 12, 2018. Accepted for publication Jul 13, 2018.

doi: $10.21037 /$ jtd.2018.07.69

View this article at: http://dx.doi.org/10.21037/jtd.2018.07.69 


\section{Introduction}

Left ventricular thrombus (LVT) is a catastrophic complication of myocardial infarction (MI), causing systemic embolism and increasing morbidity and mortality $(1,2)$. Although rapid reperfusion and potent anticoagulant therapy have reduced the incidence of LVT, it still occurs in the range of $2.5 \%$ to $15 \%$ in acute myocardial infarction (AMI) patients $(1,3)$. Left ventricular dysfunction, anterior MI, apical aneurysm and biomarkers for hypercoagulability have previously been shown as predictors of LVT in patients with AMI by previous studies $(1,2,4-8)$. However, it is still challenging to predict LVT in patients following AMI with LV dysfunction. The past decade has seen a statistically significant uptick in percutaneous coronary intervention (PCI). Currently, primary PCI for patients with AMI is the default and first-line option (9). Previous studies revealed that LVEF $<45 \%$ is an independent predictor of mortality in patients with AMI underwent primary PCI $(10,11)$. However, data regarding the occurrence and prognosis of LVT in post-MI and LV dysfunction patients who underwent PCI are rarely reported. In the current study, we analyzed the consecutive patients from a prospectively enrolled single center PCI database, with the purpose of evaluating the predictors and clinical prognosis of LVT in a high-risk subset of post-MI and LV dysfunction patients who underwent contemporary PCI treatment.

\section{Methods}

\section{Study population and design}

The Shanghai East Hospital Cardio-Invasive database prospectively enrolls all patients who received invasive procedures, including coronary intervention, ablation for arrhythmias, and interventions for congenital heart disease, since July 2012 in the Shanghai East Hospital. Detailed data on demographic and clinical characteristics, procedures, complications and clinical outcomes were prospectively documented in the computer-based database. Patient follow-up data were digitalized in the registry upon their discharge from the hospital and, then at 1, 6, and 12 months and once a year after PCI via planned clinical visits or through telephone contact by specifically trained research staff.

We screened all patients with MI (including STEMI and NSTEMI) and LV dysfunction (LVEF <45\%) who underwent PCI treatment from July 2012 to July 2016. MI was defined based on ischemic symptoms, an elevation of enzyme (CK-MB, TNT and MYO) $\geq 3$ times the upper limit of normal (ULN), with concomitant dynamic change of ECG (such as ST segment changes, new left bundle branch block, or $\mathrm{Q}$ waves) and abnormal ventricular wall motion estimated by echocardiography. Patients were excluded from the analysis if they had left ventricular ejection fraction (LVEF) $\geq 45 \%$, severe valvular disease, a history of arterial or venous thrombosis, thrombotic hematological disorders, or dilated cardiomyopathy (DCM) (Figure 1). DCM was diagnosed by heart failure symptoms, electrocardiogram and echocardiography evidence for left ventricular dilatation with reduced ejection fraction. Meanwhile, coronary angiography was performed to exclude ischemic heart disease.

In total, 903 patients in the database who had MI and underwent PCI were screened, and 636 were excluded due to the exclusion criteria. The remaining 267 were classified as patients with LVT (LVT group, $n=25$ ) or without LVT (control group, $\mathrm{n}=242$ ) (Figure 1).

This study was approved by the Institutional Review Board of Shanghai East Hospital, Tongji University. Written informed consents were obtained from all patients, and the clinical investigation was conducted according to the principle of the Declaration of Helsinki.

\section{Definition and endpoint}

In all patients, echocardiography was performed within 3 days after admission (1). LVT was defined as an echodense mass adjacent to an abnormal contracting (akinetic or dyskinetic) myocardial segment. It had to be distinguishable from the underlying endocardium, have a clear thrombusblood interface and be visible in $\geq 2$ transducer positions. LVEF was assessed according to the modified Simpson's method (12). All results were identified by at least two experienced echo physicians.

Coronary angiography and PCI were performed via femoral or radial approach according to the current guideline recommendations (13). A loading dose of aspirin $(300 \mathrm{mg})$ and clopidogrel (300 to $600 \mathrm{mg}$ ) or ticagrelor (180 mg) was given to those who underwent primary PCI, and dual-antiplatelet therapy with aspirin and clopidogrel or ticagrelor was continued for at least 12 months after the procedure for all patients. Procedural anticoagulation was achieved with preliminary administration of unfractionated heparin at a dose of 70-100 U/kg, supplemented during the procedure to maintain an activated clotting time of $>250 \mathrm{~s}$. Treatment with low molecular weight heparin (LMWH) 


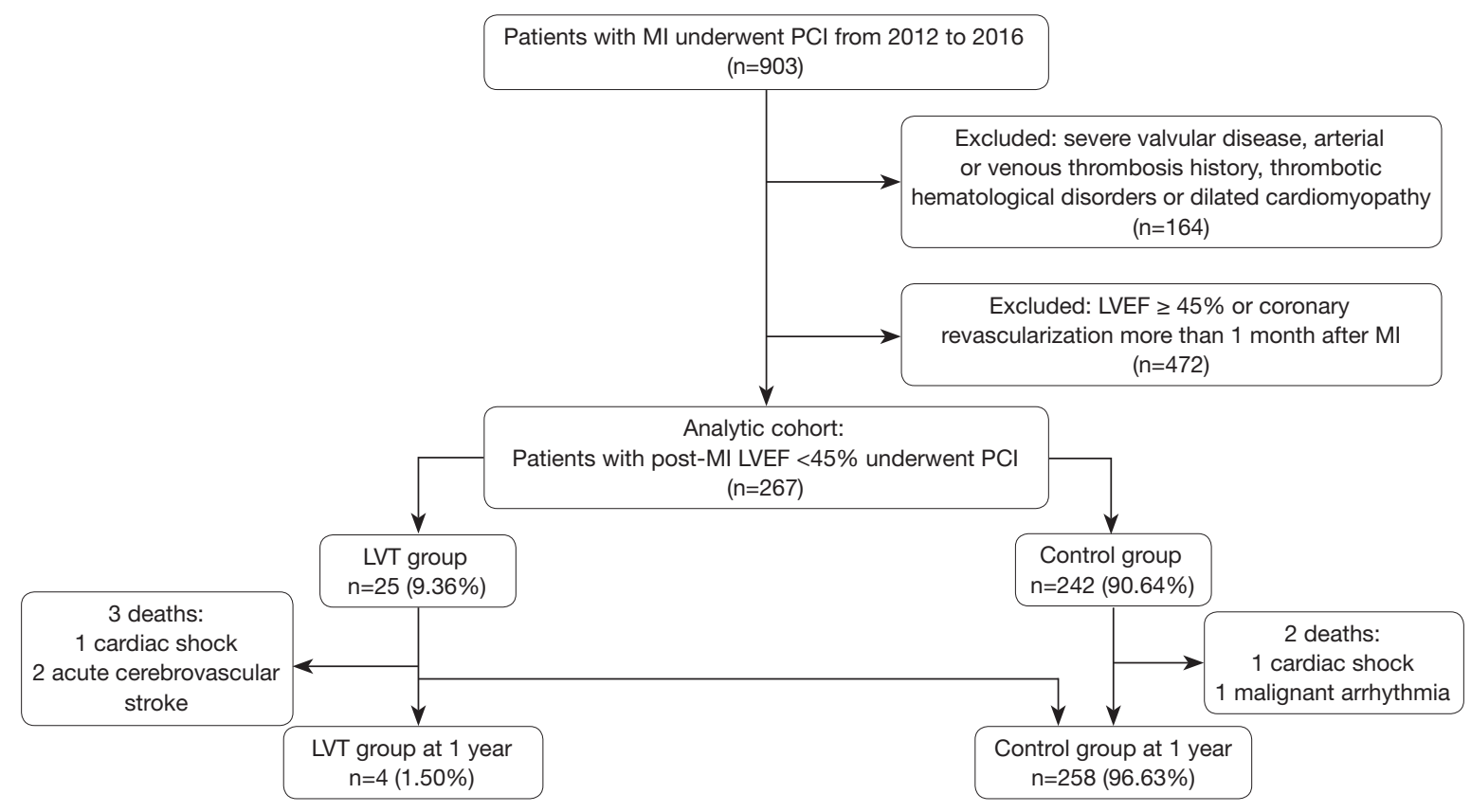

Figure 1 Study flowchart of this study. In total, 903 patients in the database who had MI and underwent PCI were screened. After excluding 636 patients due to the exclusion criteria, the remaining 267 patients were classified into the LVT group ( $\mathrm{n}=25,9.36 \%$ ) and the control group ( $\mathrm{n}=242,90.64 \%)$. The death rate was $12 \%$ vs. $0.83 \%(\mathrm{P}<0.01)$ in the LVT group compared with the Control group during follow-up. By triple anti-thrombotic therapy, the rate of LVT resolving at 1-year follow-up was $81.8 \%$.

and warfarin for patients with LVT was recommended in spite of the contraindication, aiming for a target international normalized ratio (INR) of 1.5 to 2.0 for 3 to 6 months. In addition, echocardiography was repeated for those with LVT every 3 months.

The primary endpoint of a major adverse cardiovascular and cerebrovascular event (MACCE) is defined as a composite of death, MI, target vessel revascularization (TVR) and stroke (ischemic or hemorrhagic) at 1-year clinical follow-up $(14,15)$. TVR indicates the repeated revascularization of the target vessel by PCI or coronary artery bypass grafting because of restenosis or reocclusion of the culprit lesion after PCI. Stent thrombosis was defined according to the Academic Research Consortium (ARC) definition (16). Stroke was diagnosed through the neurological examination (NIHSS) and MRI scanning, according to the guideline (15).

\section{Statistical analysis}

All of the analyses were performed using SPSS 19.0 (SPSS Inc., Chicago, IL, USA), with a nominal level of statistical significance defined as a two-tailed $\mathrm{P}<0.05$. Continuous variables are presented as the mean $\pm \mathrm{SE}$, and as frequency counts and percentages for categorical variables. The Chi-square test was used for the comparison of the nonparametric data; the $t$-test was utilized to compare the continuous variables; and the Fisher's exact test was applied to evaluate the clinical outcomes. Receiver operating characteristic (ROC) curves were plotted to predict the accuracy of the model. Logistic regression analysis was performed using a stepwise multivariable approach on the dichotomous groups. Variables with $\mathrm{P}$ values $<0.05$ were retained in the multivariable model. The odds ratio (OR) and the $95 \%$ confidence interval (CI) between the two groups were calculated. The association of LVT with time to all-cause mortality was assessed by Kaplan-Meier curves and the log-rank test.

\section{Results}

\section{Clinical characteristics, angiography, PCI and medications}

The demographic features were similar between the two groups. The results from blood examinations on admission 
were comparable between the two groups, except for the serum level of D-dimer, which was significantly higher in the LVT group. The results from the echocardiography findings showed that more patients in the LVT group had signs of LV aneurysm (Table 1).

Angiographic and PCI results were listed in Table 2. All patients in the LVT group presented with the LAD as the culprit vessel, a significantly higher SYNTAX score and a lower percentage of complete revascularization at discharge. Fewer patients in the LVT group were treated with primary PCI (60\% vs. $78.51 \%, \mathrm{P}<0.05$, Table 2).

Medications used during hospitalization were demonstrated in Table 3. There was no difference of medications except for warfarin in the two groups.

\section{Clinical outcomes}

The primary endpoint of MACCE at 1-year clinical followup was significantly higher in the LVT group $36.00 \%$ vs. $5.79 \%, \mathrm{P}<0.01)$. The cerebrovascular events and all-cause mortality were significantly increased in the LVT group $(\mathrm{P}<0.01)$. Altogether 3 patients died in the LVT group ( 1 cardiac shock and 2 hemorrhagic bleeding) and 2 patients died in the Control group (1 cardiac shock and 1 ventricular malignant arrhythmia) (Table 4, Figure 2).

During follow-up, LVT resolved in $68.2 \%$ of patients in the LVT group at the 6-month follow-up. Those with persistent LVT were continued on triple anti-thrombotic therapy, and the rate of LVT resolving at 1-year follow-up reached $81.8 \%$ (Figure 1). Dual anti-thrombotic therapy with aspirin and warfarin was recommended for those with persistent LVT.

\section{Cutoff point of the SYNTAX score, D-dimer and predictors for $L V T$}

The ROC curve analysis was performed to detect the cutoff value of the SYNTAX score and D-dimer in the prediction of LVT development. The area under the curve (AUC) values for the SYNTAX score and D-dimer were 0.91 (95\% CI: 0.86-0.97, P<0.05) and 0.83 (95\% CI: 0.75-0.91, $\mathrm{P}<0.05$ ), respectively (Figure 3). The SYNTAX score and D-dimer efficiently predict the LVT with the cutoff value of 29.50 and $1.53 \mathrm{mg} / \mathrm{L}$, respectively (Table 5).

Results from multivariate logistical regression analysis indicated that left ventricular aneurysm (OR: 1.29, 95\% CI: 1.09-1.52, $\mathrm{P}<0.01$ ), complete revascularization (OR: 0.05, 95\% CI: 0.01-0.35, P<0.01), the SYNTAX score (OR: 1.28 ,
95\% CI: $1.14-1.43, \mathrm{P}<0.01$ ) and D-dimer (OR: 1.90, 95\% CI: 1.19-3.04, $\mathrm{P}<0.01)$ were the independent predictors of LVT formations in post-MI and LV dysfunction patients in the contemporary PCI era (Figure 4).

\section{Discussion}

The results of the current retrospective analysis from a single center prospectively enrolled database indicated that the incidence of LVT in post-MI and $L V$ dysfunction patients is $9.36 \%$. The 1 -year clinical outcomes of patients with LVT were significantly worse than those without LVT, which is consistent with previous reports $(1,17)$. The presence of left ventricular aneurysm, higher SYNTAX score and serum D-dimer level were adversely related to the occurrence of LVT by multivariate analysis, while complete revascularization exerts beneficial effects in preventing LVT. The novel findings of elevated serum D-dimer level as a predictor of LVT may promote a quicker initiation of anticoagulation therapy in this high-risk subset of patients.

\section{Incidence and predictors of LVT in the contemporary primary PCI era}

The advent of primary PCI has greatly improved the clinical outcomes of STEMI patients, with significantly reduced mortality and morbidity (18-21). LVT, as one of the adverse clinical complications of STEMI, was found to be reduced in the contemporary primary PCI era, with a rapid reperfusion method and novel potent anti-thrombotic medications. Khoury et al. reported a low incidence of LVT $(1.25 \%)$ in STEMI patients treated with successful primary PCI in their cohort study (22). In a systematic review and meta-analysis, LVT was found in approximately $2.7-9.1 \%$ of post-MI patients in the contemporary PCI era (23). Heart rate, heart failure severity or reduced EF, anterior apex wall involvement, LAD disease, and non-white race were independently associated with LVT occurrence $(1,2,7)$.

In the current study, we analyzed post-MI patients with LV dysfunction who were treated with PCI, and we found that the incidence of LVT was $9.36 \%$. This incidence was higher than previous reports $(1,22)$, and this difference may be related to the high-selection of patients with LV dysfunction for the study population. Patients with baseline LV dysfunction were selected for the study population because previous studies have proved $\mathrm{LV}$ dysfunction as a major independent risk factor of LVT. We focused on these patients with the purpose of finding 
Table 1 Baseline clinical characteristics, blood examination and UCG findings between the two groups

\begin{tabular}{|c|c|c|c|}
\hline Parameters & LVT group, $\mathrm{n}=25$ & Control group, $n=242$ & $\mathrm{P}$ \\
\hline Age (y) & $66.64 \pm 2.62$ & $68.43 \pm 0.74$ & 0.46 \\
\hline Male sex & $23(92.00 \%)$ & $194(80.17 \%)$ & 0.15 \\
\hline BMI $\left(\mathrm{kg} / \mathrm{m}^{2}\right)$ & $23.13 \pm 1.51$ & $23.10 \pm 0.45$ & 0.99 \\
\hline Hypertension & $16(64.00 \%)$ & $124(51.24 \%)$ & 0.22 \\
\hline Diabetes mellitus & $6(24.00 \%)$ & $69(28.51 \%)$ & 0.63 \\
\hline Hyperlipemia & $1(4.00 \%)$ & $6(2.48)$ & 0.69 \\
\hline $\mathrm{HR}$ at presentation (bpm) & $67.30 \pm 3.99$ & $75.75 \pm 1.12$ & 0.09 \\
\hline $\mathrm{TG}(\mathrm{mg} / \mathrm{dL})$ & $1.07 \pm 0.06$ & $1.12 \pm 0.04$ & 0.66 \\
\hline $\mathrm{TC}(\mathrm{mg} / \mathrm{dL})$ & $3.97 \pm 0.24$ & $3.86 \pm 0.08$ & 0.67 \\
\hline LDL-C (mg/dL) & $2.60 \pm 1.17$ & $2.49 \pm 0.07$ & 0.65 \\
\hline $\mathrm{HDL}-\mathrm{C}(\mathrm{mg} / \mathrm{dL})$ & $1.16 \pm 0.07$ & $1.10 \pm 0.03$ & 0.50 \\
\hline Creatinine (mg/dL) & $104.59 \pm 8.29$ & $82.59 \pm 6.18$ & 0.26 \\
\hline Platelets $\left(\times 10^{9}\right)$ & $195.64 \pm 20.49$ & $206.34 \pm 5.10$ & 0.53 \\
\hline PT (s) & $13.94 \pm 0.66$ & $13.06 \pm 0.24$ & 0.27 \\
\hline Baseline INR & $1.26 \pm 0.10$ & $1.16 \pm 0.06$ & 0.58 \\
\hline CK-MB (ng/mL) & $7.39 \pm 2.17$ & $9.90 \pm 1.71$ & 0.67 \\
\hline MYO (ng/mL) & $139.43 \pm 46.67$ & $90.02 \pm 15.40$ & 0.36 \\
\hline BNP (ng/mL) & $4,898.25 \pm 1,229.07$ & $3,878.90 \pm 315.42$ & 0.33 \\
\hline \multicolumn{4}{|l|}{ Echocardiography } \\
\hline LVEDD (mm) & $55.43 \pm 2.15$ & $53.16 \pm 1.01$ & 0.30 \\
\hline LVESD (mm) & $40.77 \pm 2.30$ & $42.17 \pm 1.56$ & 0.61 \\
\hline LVEF (\%) & $38.56 \pm 1.86$ & $38.19 \pm 0.35$ & 0.85 \\
\hline LV aneurysm & $13(52.00 \%)$ & $23(9.50 \%)$ & $<0.01$ \\
\hline
\end{tabular}

BMI, body mass index; TG, triglyceride; TC, total cholesterol; LDL-C, low-density lipoprotein cholesterol; HDL-C, high-density lipoprotein cholesterol; PT, prothrombin time; FDP, fibrin degradation products; TNT, troponin T; CK-MB, creatinine kinase MB isoenzyme; MYO, myoglobin; BNP, brain natriuretic peptide; LVEDD, left ventricular end-diastolic dimension; LVESD, left ventricular end-systolic dimension; LVEF, left ventricular ejection fraction. 
Table 2 Angiographic and PCI results between the two groups

\begin{tabular}{|c|c|c|c|}
\hline Parameters & $\begin{array}{l}\text { LVT group, } \\
\quad \mathrm{n}=25\end{array}$ & $\begin{array}{l}\text { Control group, } \\
\qquad n=242\end{array}$ & $\mathrm{P}$ \\
\hline \multicolumn{4}{|l|}{ Culprit vessel distribution } \\
\hline LM & $5(20.00 \%)$ & $36(14.88 \%)$ & 0.58 \\
\hline LAD & $25(100.00 \%)$ & 209 (86.36\%) & $<0.05$ \\
\hline LCX & $13(52.00 \%)$ & $137(56.61 \%)$ & 0.80 \\
\hline $\mathrm{RCA}$ & $11(44.00 \%)$ & $147(60.74 \%)$ & 0.17 \\
\hline MVD & $9(36.00 \%)$ & 79 (32.64\%) & 0.63 \\
\hline SYNTAX score & $34.20 \pm 2.66$ & $15.26 \pm 0.49$ & $<0.01$ \\
\hline \multicolumn{4}{|l|}{$\mathrm{PCl}$ procedure } \\
\hline Primary $\mathrm{PCl}$ & $15(60.00 \%)$ & $190(78.51 \%)$ & $<0.05$ \\
\hline $\begin{array}{l}\text { The time interval from } \\
\text { diagnosis to Primary } \\
\mathrm{PCl} \text { (min) }\end{array}$ & $131.83 \pm 26.77$ & $78.81 \pm 7.49$ & $<0.05$ \\
\hline \multicolumn{4}{|l|}{ Stents } \\
\hline Number & $1.29 \pm 0.21$ & $1.45 \pm 0.06$ & 0.50 \\
\hline Diameter (mm) & $2.89 \pm 0.19$ & $2.99 \pm 0.03$ & 0.34 \\
\hline Length (mm) & $24.61 \pm 2.02$ & $26.85 \pm 0.46$ & 0.20 \\
\hline $\begin{array}{l}\text { Use of GP Ilb/IIla } \\
\text { inhibitor }\end{array}$ & $15(60 \%)$ & $112(46.3 \%)$ & 0.19 \\
\hline CR at discharge & 7 (28.00\%) & $168(69.42 \%)$ & $<0.01$ \\
\hline
\end{tabular}

LM, left main; LAD, left anterior descending coronary artery; LCX, left circumflex; RCA, right coronary artery; MVD, multivessel disease; $\mathrm{PCl}$, percutaneous coronary intervention; GP, glycoprotein; $\mathrm{CR}$, complete revascularization.

more detailed predictors of LVT. In this high-risk subset, multivariate analysis indicated that $\mathrm{LV}$ aneurysm, higher Syntax score and serum D-dimer level were independently associated with the occurrence of LVT, while complete revascularization exerts beneficial effects in preventing LVT. The SYNTAX score was first demonstrated as a predictor of LVT in patients with anterior MI treated with primary PCI with a cut-off value of 19.5 (AUC $0.697,95 \%$ CI: $0.62-0.77$, $\mathrm{P}<0.01$ ) by Gokdeniz et al. (4). In the current study, we found that a cut-off value of 29.5 for the SYNTAX score could predict the occurrence of LVT (AUC 0.91, 95\% CI: $0.86-0.97)$ with more accuracy. As a prothrombotic marker, D-dimer has been reported to be higher and to be a predictor of adverse events in patients with systolic heart failure $(24,25)$, and a higher level of D-dimer was previously found in the LVT group in a small sample study by Solheim et al. (8). Our results first demonstrated that, the serum
Table 3 Medications used during hospitalization in the two groups

\begin{tabular}{lccc}
\hline Drugs & $\begin{array}{c}\text { LVT group, } \\
\mathrm{n}=25(\%)\end{array}$ & $\begin{array}{c}\text { Control group, } \\
\mathrm{n}=242(\%)\end{array}$ & $\mathrm{P}$ \\
\hline Aspirin & $23(92.00)$ & $224(92.56)$ & 0.92 \\
Clopidogrel & $22(88.00)$ & $226(93.39)$ & 0.32 \\
Ticagrelor & $2(8.00)$ & $13(5.37)$ & 0.59 \\
Cilostazol & $1(4.00)$ & $12(4.96)$ & 0.83 \\
LMWH & $20(80.00)$ & $152(62.81)$ & 0.09 \\
Warfarin & $20(80.00)$ & $18(7.44)$ & $<0.01$ \\
Statin & $25(100.00)$ & $213(88.02)$ & 0.13 \\
ACEl/ARB & $19(76.00)$ & $151(62.40)$ & 0.18 \\
Beta blocker & $18(72.00)$ & $170(70.25)$ & 0.86 \\
CCB & $2(8.00)$ & $25(10.33)$ & 0.70 \\
Diuretic & $15(60.00)$ & $149(61.57)$ & 0.88 \\
\hline
\end{tabular}

LMWH, low molecular weight heparin; ACEI, angiotensin converting enzyme inhibitor; $\mathrm{ARB}$, angiotensin receptor blocker; $\mathrm{CCB}$, calcium channel blocker.

D-dimer level with a cut-off value of $1.53 \mathrm{mg} / \mathrm{L}$ (AUC $0.83,95 \%$ CI: $0.75-0.91)$ could independently predict the occurrence of LVT. Compared to other predictors, the serum level of D-dimer is easier to obtain in clinical setting, which may allow physicians to act at an early stage.

\section{Outcome and treatment of patients with LVT}

Patients with LVT are considered at high risk of thromboembolism $(1,26)$, and LVT is independently associated with major adverse cardiac events and with a trend toward increased mortality $(1,17,26)$. In the preprimary PCI era, LVT was associated with a historical risk of systemic embolism (SE) of approximately 20\% (27). In a recent study, in the primary PCI era, SE occurred in $16.3 \%$ of patients with LVT after first AMI during a median follow-up period of 5.4 years (26). In our study, the rate of MACCE at 1-year clinical follow-up is significantly higher in patients with LVT (36\% vs. $5.8 \%, \mathrm{P}<0.01$ ), and it is mainly attributable to the higher occurrence of ischemic stroke $(20 \%$ vs. $2.1 \%, \mathrm{P}<0.01)$.

Data from randomized trials in prevention and treatment of patients with post-MI LVT are still sparse; current recommendations from STEMI guidelines for patients with post-MI LVT include treatment with the anticoagulant warfarin with an international normalized ratio (INR) of 1.6-2.6 up to 6 months and with dual 
Table 4 Clinical outcomes in the patients

\begin{tabular}{lccc}
\hline Clinical outcomes & $\begin{array}{c}\text { LVT group, } \\
\mathrm{n}=25\end{array}$ & $\begin{array}{c}\text { Control group, } \\
\mathrm{n}=242\end{array}$ & $\mathrm{P}$ \\
\hline During hospitalization & & & \\
MACCE & $6(24.00 \%)$ & $9(3.72 \%)$ & $<0.01$ \\
Death & $2(8.00 \%)$ & $1(0.41 \%)$ & $<0.05$ \\
Reoccurrence of MI & $0(0.00 \%)$ & $1(0.41 \%)$ & 0.91 \\
TVR & $0(0.00 \%)$ & $1(0.41 \%)$ & 0.91 \\
Stroke & $4(16.00 \%)$ & $6(2.48 \%)$ & $<0.01$ \\
Ischemic & $2(8.00 \%)$ & $3(1.24 \%)$ & $<0.05$ \\
Hemorrhagic & $2(8.00 \%)$ & $3(1.24 \%)$ & $<0.05$ \\
One-year follow-up & & & \\
MACCE & $12(48.00 \%)$ & $14(5.79 \%)$ & $<0.01$ \\
Death & $3(12.00 \%)$ & $2(0.83 \%)$ & $<0.01$ \\
Reoccurrence of MI & $1(4.00 \%)$ & $2(0.83 \%)$ & 0.26 \\
TVR & $1(4.00 \%)$ & $2(0.83 \%)$ & 0.26 \\
Stroke & $7(28.00 \%)$ & $8(3.31 \%)$ & $<0.01$ \\
Ischemic & $5(20.00 \%)$ & $5(2.07 \%)$ & $<0.01$ \\
Hemorrhagic & $2(8.00 \%)$ & $3(1.24 \%)$ & $<0.05$ \\
Echocardiography & & & 0.95 \\
LVEDD (mm) & $53.00 \pm 2.08$ & $53.14 \pm 1.03$ & 0.018 \\
LVESD (mm) & $38.40 \pm 2.36$ & $42.17 \pm 1.56$ & 0.01 \\
LVEF (\%) & $38.31 \pm 2.05$ & $38.20 \pm 0.35$ & 0.94 \\
\hline
\end{tabular}

MACCE, major adverse cardiovascular and cerebrovascular event; TVR, target vessel revascularization; LVEDD, left ventricular end-diastolic dimension; LVESD, left ventricular endsystolic dimension; LVEF, left ventricular ejection fraction.

antiplatelet therapy with aspirin and clopidogrel as per ACS recommendations $(26,28,29)$. Appropriate anticoagulation therapy may decrease the incidence of embolic events without increasing the incidence of bleeding events in patients with AMI complicated by LVT (26). Ebrahimi et al. reported LVT resolution in $81.4 \%$ of patients after 6-month of combination anti-platelet and warfarin therapy (30). Cambronero-Cortinas et al. found LVT resolved in $88 \%$ patients within 1 year after anticoagulation (5). In the current study, LVT resolution was found in $68.2 \%$ and $81.8 \%$ of patients at 6 -month and 1-year clinical followup, respectively (26). Although there was no new-onset of

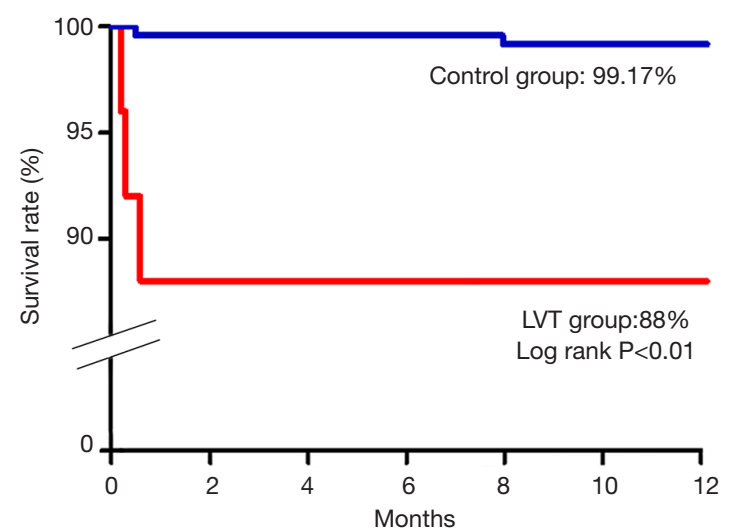

Figure 2 Survival curve of the patients. The survival rate was $88 \%$ vs. $99.17 \%(\mathrm{P}<0.01)$ in the LVT group compared with the control group.

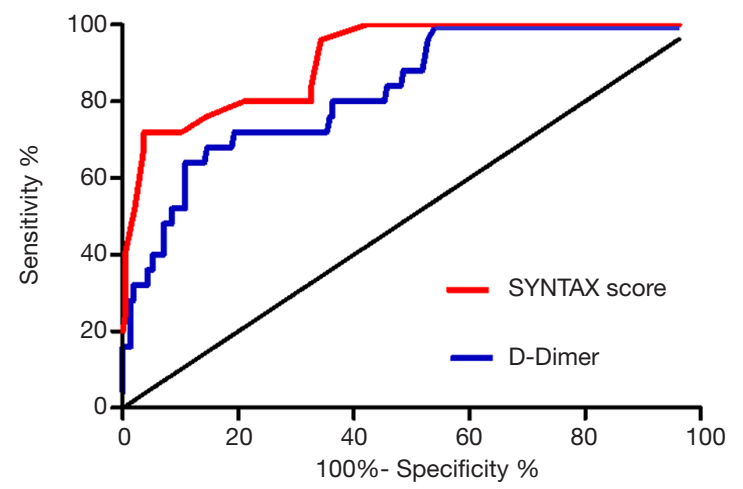

Figure 3 Receiver operating characteristic (ROC) curves of the SYNTAX score and D-dimer for identification of patients with LVT. The area under the curve (AUC) values for the SYNTAX score and D-dimer were 0.91 (95\% CI: 0.86-0.97, P<0.05) and 0.83 (95\% CI: $0.75-0.91, \mathrm{P}<0.05$ ), respectively.

hemorrhagic events in hospital survivals after discharge with triple therapy of aspirin, clopidogrel and warfarin in our study, concerns of bleeding with triple anti-thrombotic therapy still remain.

Non-fatal bleeding is an important predictor of mortality post-PCI at follow-up (31). The lower INR target of 1.5-2.0 for dual anti-platelet therapy may reduce the rate of bleeding, although this may influence the efficacy of LVT treatment. In the current study, INR was strictly controlled within the range of 1.5-2.0 during follow-up.

Prophylactic addition warfarin therapy after primary PCI in patients with apical akinesis or dyskinesis was 
Table 5 Predictive values of the parameters

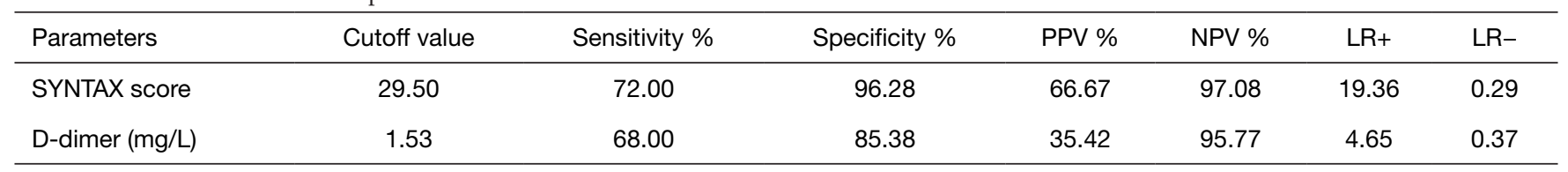

PPV, positive predictive value; NPV, negative predictive value; LR+, positive likelihood ratio; LR-, negative likelihood ratio; TNT, troponin T.

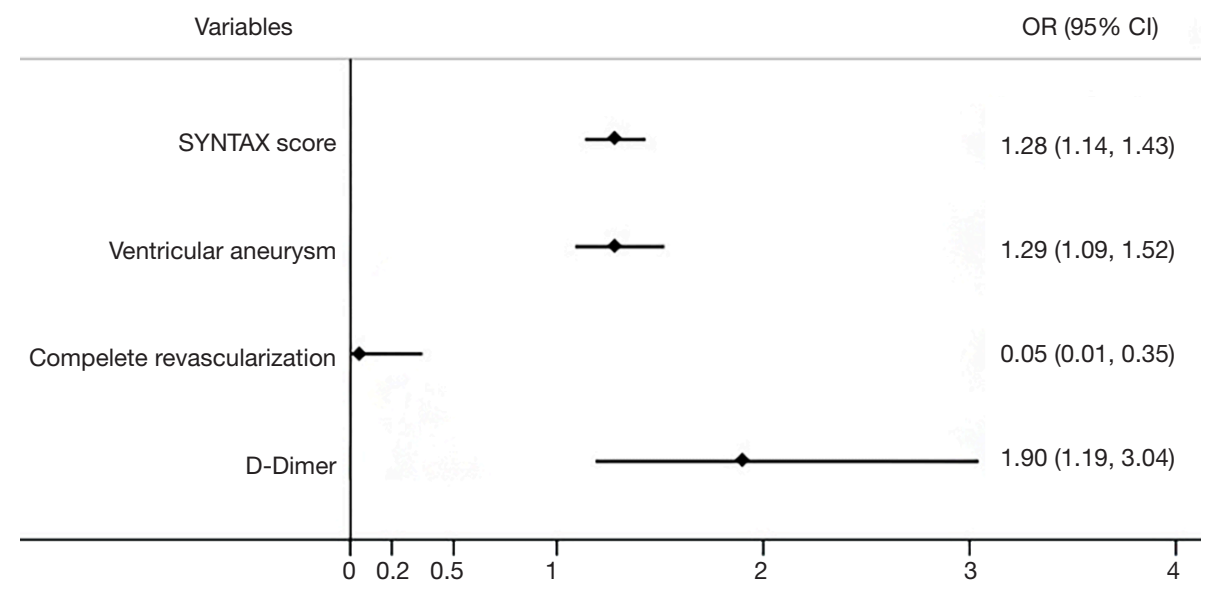

Figure 4 The forest map of the multivariate logistical regression analysis.

recommended by Le May et al. in their study (32). However, the results of a recent published meta-analysis did not show that routine use of dual anti-platelet therapy and warfarin could prevent LVT formation in anterior STEMI patients with decreased LVEF and wall motion abnormality $(33,34)$. Data on the use of non-vitamin K antagonist oral anticoagulants (NOACs) to treat or prevent LVT are limited to case reports (35). The use of NOACs instead of warfarin is still not recommended due to lack of evidence (26,36).

In conclusion, our study indicated that LVT is not uncommon in the contemporary era in patients with MI who underwent PCI. For patients with decreased LV function after MI, left ventricular aneurysm, a high SYNTAX score $(>29.5)$, incomplete revascularization and an elevated serum level of D-dimer $(>1.53 \mathrm{mg} / \mathrm{L})$ were independently related to the occurrence of LVT. These findings help to classify the high-risk LVT-prone subgroup in MI patients, and warfarin as the target prophylactic treatment may be suggested for this population.

\section{Limitations}

Our study has several limitations. First, although the data were prospectively collected, this is a single-center retrospective analysis. There is an inherent internal bias of patient selection in this study design. However, our data reflect a large real-world cohort and, therefore, may represent contemporary clinical practice. Multicenter randomized controlled trial with larger sample is expected in our future work. Second, the use of echocardiography in detecting LVT in our study had the advantage of easy use in clinical practice, but it may underestimate LVT prevalence compared with contrast-enhanced echocardiography, computed tomography (CT) or cardiac magnetic resonance imaging (MRI) $(37,38)$. Meanwhile, it would be better to exclude DCM with genetic testing or cardiac MRI. Third, the timing of performing echocardiography was varied within 3 days after admission, which may influence the detection and underestimate the rate of LVT. However, there is still no consensus regarding the timing of performing echocardiography in detecting LVT. Forth, we only enrolled patients with baseline LVEF $<0.45$, which may neglect the incidence of LVT in those with LVEF $\geq 0.45$. However, the major purpose of this study was to focus on the LVT high-risk group, which had compromised $\mathrm{LV}$ function. Finally, the first medical contact time and longer follow-up data beyond one-year were not included 
in the current study, which may substantially influence the findings of this study.

\section{Acknowledgements}

Funding: This work was supported by the National Natural Science Foundation of China (81500191, 81470392 and 81670228), Key Disciplines Group Construction Project of Pudong Health Bureau of Shanghai (PWZxq2017-05) and The National Key Research and Development Program of China (2017YFA0105600).

\section{Footnote}

Conflicts of Interest: The authors have no conflicts of interest to declare.

Ethical Statement: This study was approved by the Institutional Review Board of Shanghai East Hospital, Tongji University. Written informed consents were obtained from all patients, and the clinical investigation was conducted according to the principle of the Declaration of Helsinki.

\section{References}

1. Garber AM, Mentz RJ, Al-Khalidi HR, et al. Clinical predictors and outcomes of patients with left ventricular thrombus following ST-segment elevation myocardial infarction. J Thromb Thrombolysis 2016;41:365-73.

2. Gianstefani S, Douiri A, Delithanasis I, et al. Incidence and predictors of early left ventricular thrombus after STelevation myocardial infarction in the contemporary era of primary percutaneous coronary intervention. Am J Cardiol 2014;113:1111-6.

3. Driesman A, Hyder O, Lang C, et al. Incidence and Predictors of Left Ventricular Thrombus After Primary Percutaneous Coronary Intervention for Anterior STSegment Elevation Myocardial Infarction. Clin Cardiol 2015;38:590-7.

4. Gokdeniz T, Boyaci F, Hatem E, et al. SYNTAX score predicts the left ventricle thrombus development in patients undergoing primary percutaneous coronary intervention for first anterior myocardial infarction. Clin Appl Thromb Hemost 2014;20:698-705.

5. Cambronero-Cortinas E, Bonanad C, Monmeneu JV, et al. Incidence, Outcomes, and Predictors of Ventricular Thrombus after Reperfused ST-Segment-Elevation
Myocardial Infarction by Using Sequential Cardiac MR Imaging. Radiology 2017;284:372-80.

6. Jiang YX, Jing LD, Jia YH. Clinical Characteristics and Risk Factors of Left Ventricular Thrombus after Acute Myocardial Infarction: A Matched Case-control Study. Chin Med J (Engl) 2015;128:2415-9.

7. Shacham Y, Leshem-Rubinow E, Ben Assa E, et al. Frequency and correlates of early left ventricular thrombus formation following anterior wall acute myocardial infarction treated with primary percutaneous coronary intervention. Am J Cardiol 2013;111:667-70.

8. Solheim S, Seljeflot I, Lunde K, et al. Prothrombotic markers in patients with acute myocardial infarction and left ventricular thrombus formation treated with pci and dual antiplatelet therapy. Thromb J 2013;11:1.

9. Zhang Q, Wang XL, Liao ML, et al. Periprocedural use of tirofiban in elective percutaneous coronary intervention for long coronary lesions in stable patients with overlapping drug-eluting stents--the PETITION study: a prospective, randomized, multicenter study. Catheter Cardiovasc Interv 2015;85 Suppl 1:762-9.

10. Chew DS, Wilton SB, Kavanagh K, et al. Left ventricular ejection fraction reassessment post-myocardial infarction: Current clinical practice and determinants of adverse remodeling. Am Heart J 2018;198:91-6.

11. Kwon TG, Bae JH, Jeong MH, et al. N-terminal pro-Btype natriuretic peptide is associated with adverse shortterm clinical outcomes in patients with acute ST-elevation myocardial infarction underwent primary percutaneous coronary intervention. Int J Cardiol 2009;133:173-8.

12. Schiller NB, Shah PM, Crawford M, et al. Recommendations for quantitation of the left ventricle by two-dimensional echocardiography. American Society of Echocardiography Committee on Standards, Subcommittee on Quantitation of Two-Dimensional Echocardiograms. J Am Soc Echocardiogr 1989;2:358-67.

13. Endorsed by the Latin American Society of Interventional Cardiology; PCI WRITING COMMITTEE, Levine GN, et al. 2015 ACC/AHA/SCAI focused update on primary percutaneous coronary intervention for patients with ST-elevation myocardial Infarction: An update of the 2011 ACCF/AHA/SCAI guideline for percutaneous coronary intervention and the 2013 ACCF/AHA guideline for the management of ST-elevation myocardial infarction: A report of the American College of Cardiology/ American Heart Association Task Force on Clinical Practice Guidelines and the Society for Cardiovascular Angiography and Interventions. Catheter Cardiovasc 
Interv 2016;87:1001-19.

14. Song Y, Gao Z, Tang X, et al. Usefulness of the SYNTAX score II to validate 2 -year outcomes in patients with complex coronary artery disease undergoing percutaneous coronary intervention: A large single-center study. Catheter Cardiovasc Interv 2017. [Epub ahead of print].

15. Powers WJ, Derdeyn CP, Biller J, et al. 2015 American Heart Association/American Stroke Association Focused Update of the 2013 Guidelines for the Early Management of Patients With Acute Ischemic Stroke Regarding Endovascular Treatment: A Guideline for Healthcare Professionals From the American Heart Association/ American Stroke Association. Stroke 2015;46:3020-35.

16. Cutlip DE, Windecker S, Mehran R, et al. Clinical end points in coronary stent trials: a case for standardized definitions. Circulation 2007;115:2344-51.

17. Poss J, Desch S, Eitel C, et al. Left Ventricular Thrombus Formation After ST-Segment-Elevation Myocardial Infarction: Insights From a Cardiac Magnetic Resonance Multicenter Study. Circ Cardiovasc Imaging 2015;8:e003417.

18. Zhang Q, Zhang RY, Qiu JP, et al. One-year clinical outcome of interventionalist- versus patient-transfer strategies for primary percutaneous coronary intervention in patients with acute ST-segment elevation myocardial infarction: results from the REVERSE-STEMI study. Circ Cardiovasc Qual Outcomes 2011;4:355-62.

19. Zhang Q, Zhang RY, Zhang JS, et al. Outcomes of primary percutaneous coronary intervention for acute ST-elevation myocardial infarction in patients aged over 75 years. Chin Med J (Engl) 2006;119:1151-6.

20. Redfors B, Dworeck C, Angeras O, et al. Prognosis is similar for patients who undergo primary PCI during regular-hours and off-hours: A report from SCAAR. Catheter Cardiovasc Interv 2018;91:1240-9.

21. Satler L. Improving outcomes for primary PCI. Catheter Cardiovasc Interv 2007;69:497-9.

22. Khoury S, Carmon S, Margolis G, et al. Incidence and outcomes of early left ventricular thrombus following ST-elevation myocardial infarction treated with primary percutaneous coronary intervention. Clin Res Cardiol 2017;106:695-701.

23. Robinson AA, Jain A, Gentry M, et al. Left ventricular thrombi after STEMI in the primary PCI era: A systematic review and meta-analysis. Int J Cardiol 2016;221:554-9.

24. Zorlu A, Yilmaz MB, Yucel H, et al. Increased D-dimer levels predict cardiovascular mortality in patients with systolic heart failure. J Thromb Thrombolysis
2012;33:322-8.

25. Aispuru GR, Clavier MM, Cardone AJ, et al. Thrombotic biomarkers and left ventricle characteristics as short-term predictors of thrombotic events in patients hospitalized for acute decompensated heart failure. Eur J Intern Med 2012;23:545-51.

26. Maniwa N, Fujino M, Nakai M, et al. Anticoagulation combined with antiplatelet therapy in patients with left ventricular thrombus after first acute myocardial infarction. Eur Heart J 2018;39:201-8.

27. Lamas GA, Vaughan DE, Pfeffer MA. Left ventricular thrombus formation after first anterior wall acute myocardial infarction. Am J Cardiol 1988;62:31-5.

28. O'Gara PT, Kushner FG, Ascheim DD, et al. 2013 ACCF/ AHA guideline for the management of ST-elevation myocardial infarction: executive summary: a report of the American College of Cardiology Foundation/American Heart Association Task Force on Practice Guidelines: developed in collaboration with the American College of Emergency Physicians and Society for Cardiovascular Angiography and Interventions. Catheter Cardiovasc Interv 2013;82:E1-27.

29. Task Force on the management of STseamiotESoC, Steg PG, James SK, et al. ESC Guidelines for the management of acute myocardial infarction in patients presenting with ST-segment elevation. Eur Heart J 2012;33:2569-619.

30. Ebrahimi M, Fazlinezhad A, Alvandi-Azari M, et al. Longterm clinical outcomes of the left ventricular thrombus in patients with ST elevation anterior myocardial infarction. ARYA Atheroscler 2015;11:1-4.

31. DeEugenio D, Kolman L, DeCaro M, et al. Risk of major bleeding with concomitant dual antiplatelet therapy after percutaneous coronary intervention in patients receiving long-term warfarin therapy. Pharmacotherapy 2007;27:691-6.

32. Le May MR, Acharya S, Wells GA, et al. Prophylactic warfarin therapy after primary percutaneous coronary intervention for anterior ST-segment elevation myocardial infarction. JACC Cardiovasc Interv 2015;8:155-62.

33. Moulson N, LaHaye SA, Bertrand OF, et al. Prophylactic warfarin post anterior ST-elevation myocardial infarction: A systematic review and meta-analysis. Cardiovasc Revasc Med 2017;18:559-64.

34. Mehta SR, Bainey KR, Cantor WJ, et al. 2018 Canadian Cardiovascular Society/Canadian Association of Interventional Cardiology Focused Update of the Guidelines for the Use of Antiplatelet Therapy. Can J Cardiol 2018;34:214-33. 
35. Noflatscher M, Moes N, Gassner EM, et al. Dabigatran Added to Dual Antiplatelet Therapy to Treat a Left Ventricular Thrombus in an 87 Year Old Patient With Myocardial Infarction and Very High Bleeding Risk. Front Pharmacol 2018;9:217.

36. Habash F, Vallurupalli S. Challenges in management of left ventricular thrombus. Ther Adv Cardiovasc Dis 2017;11:203-13.

37. Meurin P, Brandao Carreira V, Dumaine R, et al. Incidence,

Cite this article as: You $\mathrm{J}$, Wang $\mathrm{X}, \mathrm{Wu} \mathrm{J}$, Gao L, Wang X, Du P, Liu H, Li J, Wang Y, Liang Y, Guo W, Zhang Q. Predictors and prognosis of left ventricular thrombus in postmyocardial infarction patients with left ventricular dysfunction after percutaneous coronary intervention. J Thorac Dis 2018;10(8):4912-4922. doi: 10.21037/jtd.2018.07.69 diagnostic methods, and evolution of left ventricular thrombus in patients with anterior myocardial infarction and low left ventricular ejection fraction: a prospective multicenter study. Am Heart J 2015;170:256-62.

38. Gellen B, Biere L, Logeart D, et al. Timing of Cardiac Magnetic Resonance Imaging Impacts on the Detection Rate of Left Ventricular Thrombus After Myocardial Infarction. JACC Cardiovasc Imaging 2017;10:1404-5. 